Pacific Journal of Mathematics

TECHNIQUE FOR THE DETECTION OF OSCILLATION OF 


\section{A TECHNIQUE FOR THE DETECTION OF OSCILLATION OF SECOND ORDER ORDINARY DIFFERENTIAL EQUATIONS}

\section{KoMkov}

An iterative procedure is used to determine the oscillatory properties of second order linear differential equations, using a repeated application of the Kummer-Liouville transformation.

1. The Kummer-Liouville transformation. We shall consider the oscillatory properties of the equation

$$
\left(a(t) x^{\prime}\right)^{\prime}+c(t) x=0 \quad\left({ }^{\prime}=\frac{d}{d t}\right)
$$

$$
\begin{aligned}
& a(t) \in C^{1}\left[t_{0}, \infty\right), a(t)>0, \\
& c(t) \in C\left[t_{0}, \infty\right) .
\end{aligned}
$$

Let $\varphi(t) \in C^{1}\left[t_{0}, \infty\right), \varphi^{\prime}(t)>0, \lim _{t \rightarrow \infty} \varphi(t)=\infty$,

$$
\psi(t) \in C^{2}\left[t_{0}, \infty\right), \psi(t) \neq 0, \quad t_{0} \leqq t<\infty .
$$

It was shown by Kummer ([5], 1834) that the transformation $\tau=$ $\varphi(t), x(t)=\psi(t) y(\tau)$ transforms the equation (1) into an equation of the same form:

$$
\left(R(\tau) y^{\prime}(\tau)\right)^{\prime}+Q(\tau) y(\tau)=0
$$

The formulas for $R(\tau), Q(\tau)$ are:

$$
\begin{aligned}
& R(\tau(t))=a(t) \varphi^{\prime}(t) \psi^{2}(t) \\
& Q(\tau(t))=\left[\left(a(t) \psi^{\prime}(t)\right)^{\prime}+c(t) \psi(t)\left[\varphi^{\prime}(t)\right]^{-1}\right] \psi(t),
\end{aligned}
$$

(see [5], or the expository article [7]). Moreover, if $\varphi(t)$ is chosen to be

$$
\varphi(t)=\int_{t_{0}}^{t}\left[\alpha(\xi) \psi^{2}(\xi)\right]^{-1} d \xi,
$$

then the equation $\left(1^{a}\right)$ assumes the form

$$
y^{\prime \prime}(\tau)+\sigma(\tau) y(\tau)=0
$$

In the specific case when $\int_{t}^{\infty}[a(\xi)]^{-1} d \xi=\infty$, the choice $\psi(t) \equiv 1$ results in the formula 


$$
\sigma(\tau)=a(t) c(t) \quad \text { (This was known to Liouville) . }
$$

For the case when $\int_{t_{0}}^{\infty}[a(\xi)]^{-1} d \xi<\infty$, it is convenient to choose

$$
\begin{gathered}
\psi(t)=\int_{t}^{\infty}[a(\xi)]^{-1} d \xi \\
\varphi(t)=\int_{t_{0}}^{t}\left[a(\xi) \psi^{2}(\xi)\right]^{-1} d \xi .
\end{gathered}
$$

With the above choices the equation (2) is oscillatory if and only if the equation (1) is oscillatory.

2. An iteration of the Kummer-Liouville transformation. We introduce a transformation

$$
y(\tau)=\eta(\tau) \exp \left(\Phi_{1}(\tau)\right)
$$

where $\Phi_{1}(\tau)$ is a real valued function of the class $C^{2}$ on $\left[t_{0}, \infty\right)$. Obviously the zeros of $y(\tau)$ and of $\eta(\tau)$ will coincide on $\left[t_{0}, \infty\right)$. We compute

$$
y^{\prime \prime}=\left\{\left[\Phi_{1}^{\prime \prime}+\left(\Phi_{1}^{\prime}\right)^{2}\right] \eta+2 \Phi_{1}^{\prime} \eta^{\prime}+\eta^{\prime \prime}\right\} \exp \left(\Phi_{1}\right)
$$

and we have

$$
\begin{aligned}
& \quad \exp \left(\Phi_{1}(\tau)\right)\left[y^{\prime \prime}(\tau)+\sigma(\tau) y(\tau)\right]= \\
& \quad\left(\exp \left(2 \Phi_{1}(\tau)\right) \eta^{\prime}\right)^{\prime}+\exp 2 \Phi_{1}(\tau)\left[\sigma(\sigma)+\Phi_{1}^{\prime \prime}+\left(\Phi_{1}^{\prime}\right)^{2}\right] \eta(\tau)=0 .
\end{aligned}
$$

The equation (8) is again of the same type as the equation (1) and we can again apply the Kummer-Liouville transformation. There are two possibilities.

$$
\begin{gathered}
\int_{t_{0}}^{\infty} \exp \left(-2 \Phi_{1}(\xi)\right) d \xi=+\infty \\
\int_{t_{0}}^{\infty} \exp (-2 \Phi(\xi)) d \xi<\infty .
\end{gathered}
$$

We shall only consider in detail the case $\left(^{*}\right)$, that is, we choose $\Phi_{1}(\xi)$ so that the condition $\left(^{*}\right)$ is satisfied. It suffices if $\Phi(\xi)$ is bounded above on some ray $\left[\xi_{0},+\infty\right)$.

The transformation

$$
\tau_{1}=\varphi_{1}(\tau)=\int_{t_{0}}^{\tau} \exp \left(-2 \Phi_{1}(\xi)\right) d \xi, \quad \psi(\tau) \equiv 1,
$$

then changes the equation (8) again into the equation of the form (2) (We shall refer to this specific form of Kummer transformation as the Kummer-Liouville transformation). 


$$
\begin{gathered}
y_{1}^{\prime \prime}\left(\tau_{1}\right)+\sigma_{1}\left(\tau_{1}\right) y\left(\tau_{1}\right)=0, \quad \text { where } \\
\sigma_{1}\left(\tau_{1}(\tau)\right)=\exp \left(4 \Phi_{1}(\tau)\right)\left[\sigma(\tau)+\Phi_{1}^{\prime \prime}(\tau)+\left(\Phi^{\prime}(\tau)\right)^{2}\right] .
\end{gathered}
$$

We observe that the inverse function $\varphi_{1}^{-1}: \tau_{1} \rightarrow \tau$ is defined on $[0, \infty)$, since $\varphi_{1}$ was monotone increasing, mapping $\left[t_{0}, \infty\right)$ onto $[0, \infty)$, and $\sigma_{1}\left(\tau_{1}\right)$ can be written in explicit form if the variable $\tau$ is replaced by $\varphi^{-1}\left(\tau_{1}\right)$ on the right hand side of the equality (9).

Since the equation $\left(2^{a}\right)$ has the same oscillatory properties as (2), this can be used to derive new criteria of oscillation, or non-oscillation from a known oscillatory criterion.

As an example we shall derive the Leighton criterion that equation (2) is oscillatory if

$$
\int_{1}^{\infty}\left(t \sigma(t)-\frac{1}{4 t}\right) d t=\infty
$$

from the Leighton-Wintner criterion

$$
\int_{t}^{\infty} \sigma(t) d t=+\infty
$$

EXAMPLE 1. We choose $\Phi_{1}(t)=\Lambda \log t, t>1, \Lambda \leqq 1 / 2$.

$$
\sigma_{1}(t)=t^{4} \Lambda \sigma(t)-\Lambda(1-\Lambda) t^{41-2} \text {. }
$$

The transformation of variable

$$
\tau= \begin{cases}\frac{t^{-2 \Lambda+1}}{-2 \Lambda+1} & \Lambda<\frac{1}{2}, \\ \log t & \Lambda=\frac{1}{2},\end{cases}
$$

gives in the particular case $\Lambda=1 / 2$

$$
\sigma_{1}(\tau)=e^{2 \tau} \sigma\left(e^{\tau}\right)-\frac{1}{4}
$$

The Leighton-Wintner condition becomes

$$
\int^{\infty}\left[\xi \sigma(\xi)-\frac{1}{4 \xi}\right] d \xi=+\infty,
$$

which is Leighton's condition.

If on the other hand we choose $\Lambda=1 / 4$, we obtain the following sufficient condition for oscillation of (2):

$$
\int^{\infty}\left(t^{1 / 2} \sigma(t)-\frac{3}{16} t^{-1(1 / 2)}\right) d t=+\infty .
$$


Obviously one can experiment with other choices of the function $\Phi_{1}(t)$ and with other necessary, or sufficient conditions for oscillation of (2).

With the same choice of $\Phi_{1}(t)=\Lambda \log t, 0<\Lambda \leqq 1 / 2$, we have for example the following generalization of Wintner's criterion:

$$
\lim _{t \rightarrow \infty} t^{-1} \int\left[\int^{\eta} \sigma(\xi) d \xi\right] d \eta=+\infty
$$

If for any $\Lambda<1 / 2$,

$$
\lim _{t \rightarrow \infty} t^{-1} \int^{t}\left\{\int^{\eta}\left[\tau^{2.1} \sigma(\tau)-\Lambda(1-\Lambda) \tau^{2.1-2}\right] d \tau\right\} d \eta=+\infty
$$

then the solutions of (2) are oscillatory.

EXAMPLE 2. (An alternate form of Wong's theorem). In [9] Wong has shown that a necessary and sufficient condition for the existence of a bounded oscillatory solution of (2) is:

$\int^{\infty} t \sigma(t) d t=+\infty ; \quad$ (see also [16], and [6] for generalization to

higher order equations) .

Since boundedness is not necessarily preserved by the Kummer transformation, we shall only use the sufficiency condition for non-oscillation known to Bôcher [2].

$$
\int^{\infty} t \sigma(t) d t<\infty \quad \text { (also known as Kondrat'ev's lemma). }
$$

Substituting as before

$$
\begin{gathered}
\Phi_{1}(t)=\Lambda \log t, \quad \Lambda=\frac{1}{2}, \quad \text { we obtain } \\
\int^{\infty} \tau \sigma_{1}(\tau) d \tau=\int^{\infty} \tau\left(e^{2 \tau} \sigma\left(e^{\tau}\right)-\frac{1}{4}\right) d \tau=\int^{\infty} \log \xi\left(\xi^{2} \sigma(\xi)-\frac{1}{4}\right) \frac{1}{\xi} d \xi \\
=\int_{t_{0}}^{\infty} \log t\left(t \sigma(t)-\frac{1}{4 t}\right) d t<\infty, \quad t_{0} \geqq 1,
\end{gathered}
$$

as a sufficient condition for non-oscillation of (2), which is of interest on its own merits.

Clearly a great variety of new necessary, or sufficient conditions could be generated from known conditions for oscillatory behavior of solutions. We shall not pursue this much farther, but will conclude this section by offering one more example.

We consider the equations:

$$
y^{\prime \prime}+\sin t y=0
$$


(b)

$$
y^{\prime \prime}+\left[\sin ^{2} t \cos (\cos t)-\cos t \sin (\cos t)\right] y=0 .
$$

The equation (a) is known to be oscillatory. A proof of the oscillatory behavior of (a) was originally given by in 1946 (using a Fourier series expansion of the solution); see [11], however it is easily settled by a result of Hartman of 1952, [4]. A proof of oscillation of (b) is not immediately obvious. Both are easily given if one chooses $\Phi_{1}(t)=$ $\sin t$ for (a) and $\Phi_{1}(t)=\cos (\cos t)$ for $(b)$.

We have in the case of (a)

$$
\begin{aligned}
\int^{\infty} \sigma_{1}(t) d \tau & =\int^{\infty}[\exp (4 \sin t)] \cos ^{2} t \frac{d \tau}{d t} d t \\
& =\int^{\infty}[\exp (2 \sin t)] \cos ^{2} t d t=+\infty,
\end{aligned}
$$

since $\int_{d}^{d+2 \pi} \sigma_{1}(t) d \tau$ is a positive number independent of $d$ on any interval $[d, d+2 \pi]$. This proves the oscillatory behavior of (a).

In this case of equation (b), we have chosen:

$$
\begin{aligned}
\Phi(t) & =\cos (\cos t) \\
\left(\Phi^{\prime}\right)^{2}+\Phi^{\prime \prime}+\sigma(t) & =\sin ^{2} t \sin ^{2}(\cos t)
\end{aligned}
$$

and it is clear that:

$$
\int_{\pi / 2}^{\infty}[\exp (2 \cos (\cos t))]\left[\sin ^{2} t \sin ^{2}(\cos t)\right] d t=+\infty
$$

for exactly the same reasons as in the case (a).

2. An iterative procedure. Since equation $\left(1^{a}\right)$ and (1) are identical in form, the Kummer transformation can be iterated any number of times. Using at each step the additional transformation (6), i.e. $y\left(\tau_{i}\right)=\eta\left(\tau_{i}\right) \exp \left(\Phi_{i+1}\left(\tau_{i}\right)\right)$, we obtain a sequence of equations of the form:

$$
y_{i}^{\prime \prime}\left(\tau_{i}\right)+\sigma_{i}\left(\tau_{i}\right) y_{i}\left(\tau_{i}\right)=0
$$

where

$$
\left.\sigma_{i}\left(\tau_{i}\left(\tau_{i-1}\right)\right)=\exp \left(4 \Phi_{i}\left(\tau_{i-1}\right)\right)\left[\sigma\left(\tau_{i-1}\right)\right)+\Phi_{i}^{\prime \prime}\left(\tau_{i-1}\right)+\left(\Phi_{i}^{\prime}\right)^{2}\left(\tau_{i-1}\right)\right] .
$$

If the condition $\left(^{*}\right)$ is obeyed by each choice of $\Phi_{i}(\tau), i=1,2, \cdots, k$, we arrive at a sequence of transformations, with new variables $\tau_{i}$ defined by

$$
\tau_{i}=\int_{c}^{\pi_{i-1}} \exp \left(-2 \Phi_{i}(\xi)\right) d \xi=\varphi_{i}\left(\tau_{i-1}\right),
$$

where $c$ is a suitably chosen constant. It would appear at a first glance that litte could be gained by such iterative methods, since a. 
product of two Kummer-Liouville transformations is again a KummerLiouville transformation, and in fact

$$
\tau_{i}=\varphi_{i}\left(\tau_{i-1}\right)=\varphi_{i}\left(\varphi_{i-1}\left(\tau_{i-2}\right)\right),
$$

can be replaced by a single transformation of the same kind. This does not mean that such a procedure is not of practical use for "improving" in some sense the oscillatory properties of a given second order equation. A meaning of the term "improving" will be given later in $\S 3$ of this paper, when we define the "speeding up" of oscillation. At this point we shall only show that new formulas are obtained by iterating twice, even if the same function $\Phi_{1}(\xi)=\Phi_{2}(\xi)$ is used in consecutive steps, and that indeed there are equations for which the second iterative formula gives an easily applicable sufficient condition for oscillation, while the formula obtained in the first iteration, and the original criterion were inconclusive. Moreover, the choice of the function $\Phi_{i}\left(\tau_{i-1}\right)$ at each step was routine, while the choice of a function $\varphi_{i}\left(\tau_{i-2}\right)$ is very complex.

Let us as an example iterate once more the Leighton-Wintner criterion, using the same choice of function $\Phi_{1}(t)=(1 / 2) \log t, \Phi_{2}\left(\tau_{1}\right)=$ $(1 / 2) \log \tau_{1}$. After the first iteration we had:

$$
\begin{aligned}
\sigma_{1}(t) & =\exp \left(4 \Phi_{1}(t)\right)\left[\sigma_{0}(t)+\left(\Phi^{\prime}(t)\right)^{2}+\Phi^{\prime \prime}(t)\right] \\
\text { with } \quad \tau_{1} & =\int \exp \left(-2 \Phi_{1}(\xi)\right) d \xi, \quad\left(\sigma_{0}(t) \equiv \sigma(t)\right) .
\end{aligned}
$$

In this case with the choice $\Phi_{1}(t)=(1 / 2) \log t$, and $\tau_{1}=\log t$, we have

$$
\sigma_{1}\left(\tau_{1}\right)=\exp \left(2 \tau_{1}\right)\left[\sigma_{0}\left(e^{\tau_{1}}\right)-\frac{1}{4} e^{-2 \tau_{1}}\right] .
$$

Using again the same choice $\Phi_{2}\left(\tau_{1}\right)=(1 / 2) \log \tau_{1}$, we obtain a second iterative function

$$
\sigma_{2}\left(\tau_{1}\right)=\tau_{1}^{2}\left\{\exp \left(2 \tau_{1}\right)\left[\sigma_{0}\left(e^{\tau_{1}}\right)-\frac{1}{4} e^{-2 \tau_{1}}\right]-\frac{1}{4} \tau_{1}^{2}\right\} .
$$

The Leighton-Wintner criterion can be rewritten in the following form:

$$
\begin{aligned}
\int^{\infty} \sigma_{2}\left(\tau_{1}\right) \frac{d \tau_{2}}{d \tau_{1}} d \tau_{1} & =\int^{\infty} \tau_{1}\left\{\exp \left(2 \tau_{1}\right)\left[\sigma_{0}\left(e^{\tau_{1}}\right)-\frac{1}{4} e^{-2 \tau_{1}}\right]-\frac{1}{4} \tau_{1}^{2}\right\} d \tau_{1} \\
& =+\infty
\end{aligned}
$$

A substitution $\xi=e^{\tau_{1}}$ results in the following sufficient condition for oscillation: 
(F)

$$
\int^{\infty} \frac{\log \xi}{\xi}\left\{\left[\xi^{2} \sigma_{0}(\xi)-\frac{1}{4}\right]-\frac{1}{4 \log ^{2} \xi}\right\} d \xi=+\infty .
$$

As an example of application we consider the equation

$$
y^{\prime \prime}+\frac{1}{4 t^{2}}\left(1+\frac{\alpha}{\log ^{2} t}\right) y=0, \quad \alpha>0 .
$$

Application of condition (F) confirms that solutions of this equation are oscillatory if $\alpha>1$. (It is known that this equation is non-oscillatory if $\alpha<1 / 4$.)

Further interesting criteria are easily developed by other iteration of formulas (10) and (11) with other choices of functions $\Phi_{i}\left(\tau_{i-1}\right)$. This technique is summarized in theorems 1 and 2.

THEOREM 1. Let $\left\{\Phi_{i}(\xi)\right\}$ be a sequence of functions such that each function $\Phi_{i}(\xi)$ is of the class $C^{2}$ on some infinite ray $\left[\hat{\xi}_{i 0},+\infty\right]$, and has the property

$$
\int_{\xi i 0}^{\infty} \exp \left(-2 \Phi_{i}(\xi)\right) d \xi=+\infty
$$

then the equation

$$
x^{\prime \prime}(t)+\sigma_{0}(t) x(t)=0
$$

is oscillatory if and only if the equation

$$
y_{i}^{\prime \prime}\left(\tau_{i}\right)+\sigma_{i}\left(\tau_{i}\right) y_{i}\left(\tau_{i}\right)=0
$$

is oscillatory, where $\sigma_{i}$, $\tau_{i}$ are determined recursively by the transformations (12) and (13) given below.

(Note: In the case where $\int^{\infty} \exp \left(-2 \Phi_{i}\right) d \xi<\infty$, the alternative formulas (14), (15) are available.)

$$
\begin{gathered}
\sigma_{i}\left(\tau_{i-1}\right)=\exp \left\{4 \Phi_{i}\left(\tau_{i-1}\right)\right\}\left[\sigma_{i-1}\left(\tau_{i-1}\right)+\left(\Phi_{i}^{\prime}\left(\tau_{i-1}\right)\right)^{2}+\Phi_{i}^{\prime \prime}\left(\tau_{i}\right)\right], \\
\tau_{i}=\varphi_{i}\left(\tau_{i-1}\right)=\int_{\xi_{0}}^{\tau_{i-1}} \exp \left(-2 \Phi_{i}(\xi)\right) d \xi .
\end{gathered}
$$

We use here the notation:

$$
\left\{\begin{array}{l}
\Phi_{i}^{\prime}\left(\tau_{i-1}\right) \equiv \frac{d}{d \tau_{i-1}} \Phi_{i}\left(\tau_{i-1}\right) \\
\Phi_{i}^{\prime \prime}\left(\tau_{i-1}\right) \equiv \frac{d^{2}}{d \tau_{i-1}^{2}} \Phi_{i}\left(\tau_{i-1}\right), \quad \text { etc . }
\end{array}\right.
$$

It is clear that any oscillation, or non-oscillation tests applied to $\left(2^{b}\right)$ will be valid tests for solutions of $\left(2^{a}\right)$. 
As an example of application of this theorem we prove that the solutions of Mathieu equation

$$
x^{\prime \prime}(t)+(\alpha+\beta \sin t) x=0
$$

are oscillatory if $\alpha>-\left(\beta^{2} / 2\right)$.

We introduce $\Phi_{1}(t)=\beta \sin t$.

Hence

$$
\begin{aligned}
& \sigma_{1}(t)=\exp (4 \beta \sin t)\left[\alpha+\beta \sin t+\beta^{2} \cos ^{2} t-\beta \sin t\right] \\
& \quad=\exp (4 \beta \sin t)\left[\alpha+\beta^{2} \cos ^{2} t\right] \\
& \int^{\infty} \sigma_{1}(t) \frac{d \tau_{1}}{d t} d t=\int^{\infty} \exp (2 \beta \sin t)\left[\alpha+\beta^{2} \cos ^{2} t\right] d t .
\end{aligned}
$$

However if $\alpha>-\left(\beta^{2} / 2\right)$, then

$$
\begin{gathered}
\int_{d}^{d+\pi}\left(\alpha+\beta^{2} \cos ^{2} t\right) d t>0, \text { and } \\
\int_{d}^{d+\pi}\left\{\exp (2 \beta \sin t)\left[\alpha+\beta^{2} \cos ^{2} t\right]\right\} d t=C,
\end{gathered}
$$

where $C$ is a constant independent of $d$. Hence the transformed equation

$$
y^{\prime \prime}(\tau)+\sigma_{1}(\tau) y(\tau)=0
$$

is oscillatory.

(See the 1963 article of Zubova [12] for a discussion of more detailed results concerning the Mathieu equation.)

There are many ways of interpreting Theorem 1. For example, we can restate Theorem 1 in the following way.

Theorem $1^{a}$. If there exists a $C^{2}$ function $f(t)>0, t \in\left[t_{0}, \infty\right)$, such that $\int^{\infty} f^{-1}(t) d t=\infty$ and if

$$
\int^{\infty}\left\{\sigma f-\frac{\left(f^{\prime}\right)^{2}}{4 f}+\frac{f^{\prime \prime}}{2}\right\} d t=+\infty,
$$

then the solutions of (2) are oscillatory.

This result is obtained immediately by identifying

$$
\Phi_{1}(t)=\frac{1}{2} \log f(t) \text {. }
$$

An application of this result to detect oscillation in the case when $\sigma(t)>0$ for sufficiently large values of $t$, and $\int^{\infty} \sigma(t) d t<\infty$, is obtained 
by putting $f(t)=1 / \sigma$ and observing that for sufficiently large values of $t, \log 1 / \sigma$, and $(\log 1 / \sigma)^{-1}$ is defined. Hence on some ray $[\hat{t}, \infty)$ we have the condition:

$$
\int^{\infty}\left(1-\frac{\sigma^{\prime}}{\sigma^{3}}-\frac{\sigma^{\prime \prime} \sigma-2\left(\sigma^{\prime}\right)^{2}}{2 \sigma^{3}}\right) d t=+\infty
$$

as the sufficient condition for oscillation. Since there seems to be no immediate application of such criteria, we shall not pursue this line of reasoning any further.

3. The "speeding up" and "slowing down" of oscillations. We consider the transformation $T: \sigma_{i} \rightarrow \sigma_{i+1}$ as defined by formulas (12) and (13), and the representation $\sigma_{i+1}\left(\tau_{i+1}\right)=\sigma_{i+1}\left(\tau_{i+1}\left(\tau_{i}\right)\right)$.

It is clear that in the cases when we suspect oscillatory behavior we are interested in such transformations with the property

$$
\frac{d_{\tau_{i+1}} \sigma_{i+1}}{d_{\tau_{i}}}\left(\tau_{i+1}\left(\tau_{i}\right)\right)>\sigma_{i}\left(\tau_{i}\right)
$$

for all (almost all) $\tau_{i}$ on some ray $\tau_{i}$ on some ray $\tau_{i} \in[\hat{\tau}, \infty)$. On the other hand if we wish to demonstrate that the equation is disconjugate beyond some point $\hat{\tau}$, we are interested in transformations which reverse the above inequality, i.e. we want the transformation to have the property

$$
\frac{d_{\tau_{i+1}}}{d_{\tau_{i}}} \sigma_{i+1}\left(\tau_{i+1}\left(\tau_{i}\right)\right)<\sigma_{i}\left(\tau_{i}\right) \text { on some infinite ray }[\hat{\tau}, \infty) .
$$

This is equivalent to the statement, that we choose $\Phi$ such that

$$
\begin{array}{ll}
\exp (2 \Phi)\left[\sigma+\left(\Phi^{\prime}\right)^{2}+\Phi^{\prime \prime}\right]-\sigma>0 & \text { in the first case, and } \\
\exp (2 \Phi)\left[\sigma+\left(\Phi^{\prime}\right)^{2}+\Phi^{\prime \prime}\right]-\sigma<0 & \text { in the second case }
\end{array}
$$

on some ray $\left[t_{0}, \infty\right)$. We shall say that these transformations "speed up" or "slow down" respectively the oscillation of (2).

Looking at the second inequality, and rewriting it in the form

$$
\left(\Phi^{\prime}\right)^{2}+\Phi^{\prime \prime}+\sigma-\sigma \exp (-2 \Phi)<0
$$

we can regard it as Ricatti's inequality in the variable $\Phi^{\prime}=u$ :

$$
u^{2}+u^{\prime}+p(t)<0, \text { where } p(t)=\sigma[1-\exp (-2 \Phi(t))] .
$$

This inequality can be satisfied on $\left[t_{0}, \infty\right)$ by some $u=\Phi^{\prime}$ only if the equation $y^{\prime \prime}+\sigma(1-\exp (-2 \Phi)) y=0$ is disconjugate on $\left[t_{0}, \infty\right)$.

ExAMPLE. The choice of $\Phi(t)=(1 / 2) \alpha \log t$ can result in slowing 
down of oscillation only if the equation $y^{\prime \prime}+\sigma\left(1-t^{-1}\right) y=0$ is disconjugate on some ray $\left[t_{0}, \infty\right)$.

The following theorem is an immediate consequence of our discussion.

THEOREM 2. Let us assume that the equation (2), i.e. $y^{\prime \prime}+\sigma(t) y=$ 0 is disconjugate on some ray $\left[t_{0}, \infty\right)$, on which $\sigma(t)$ is positive. Then there exists a function $\Phi(t) \in C^{2}\left[t_{0}, \infty\right)$ such that a transformation determined by $\Phi(t)$ and defined by formulas (12), (13), or alternatively by formulas (14), (15) below, slows down the oscillation of (2).

Proof. Let us assume that the equation (2) is disconjugate on $\left[t_{0}, \infty\right)$, and $\sigma(t)>0$ on $\left[t_{0}, \infty\right)$. Then there exists a function $u \in$ $C^{1}\left[t_{0}, \infty\right)$, such that $u^{\prime}+u^{2}+\sigma(t) \leqq 0$ for all $t \in\left[t_{0}, \infty\right)$. Hence it is possible to find a function $\Phi(t) \in C^{2}\left[t_{0}, \infty\right)$ such that $\left(\Phi^{\prime}\right)^{2}+\Phi^{\prime \prime}+\sigma(t) \leqq$ $0, t \in\left[t_{0}, \infty\right)$. Let us assume that

$$
\int^{\infty} \exp (-2 \Phi) d t=+\infty \text {. }
$$

Then the transformation determined by $\Phi(t)$ as defined by (12) and (13) will "slow down" the oscillation of equation (2). If this statement was false, then on some subinterval of $\left[t_{0}, \infty\right)$ we would have

$$
\left(\Phi^{\prime}\right)^{2}+\Phi^{\prime \prime}+\sigma(1-\exp (2 \Phi)) \geqq 0,
$$

implying that on this subinterval $-\sigma(t) \exp (-2 \Phi(t)) \geqq 0$, which is impossible.

In the case $\int^{\infty} \exp (-2 \Phi) d t<\infty$ (which is the only remaining case), the Kummer transformation is determined by the formulas

$$
\begin{gathered}
\tau_{i+1}=\int_{\tau_{i}}^{\infty} \exp (-2 \Phi(\xi)) d \xi=\psi\left(\tau_{i}\right) \\
\sigma_{n+1}\left(\tau_{i}\right)=\exp \left(4 \Phi\left(\tau_{i}\right)\right)\left[\sigma_{n}\left(\tau_{i}\right)+\Phi^{\prime \prime}+\left(\Phi^{\prime}\right)^{2}\right] \psi^{4}\left(\tau_{i}\right) .
\end{gathered}
$$

Again the denial of the condition of "slowing down" of the oscillation involves the inequality

$$
\left(\Phi^{\prime}\right)^{2}+\Phi^{\prime \prime}+\sigma\left(1-\psi^{-4} \exp (-2 \Phi)\right) \geqq 0
$$

which by a repetition of previous arguments would imply

$$
-\sigma \psi^{4} \exp (-2 \Phi) \geqq 0
$$

on some subinterval on which $\sigma(t)>0$, which again is impossible. This concludes the proof.

Note. The referee has pointed out that the idea of iterating the 
Kummer-Liouville transformation is not new, and is in fact proposed in the book of R. Bellman [1], pp. 120-122.

\section{REFERENCES}

1. R. Bellman, Stability Theory for Ordinary Differential Equations, McGraw-Hill, New York, 1953

2. M. Bôcher, The theorem of oscillation of Sturm and Klein, I, II, Bull. Amer. Math. Soc., 4 (1897), 295-313 and 365-376.

3. M. I. Elŝin, On a solution of a classical oscillation problem, Dokl. Akad. Nauk SSSR, 68 (1949), 221-224.

4. P. Hartman, On non-oscillatory linear differential equations of second order, Amer. J. of Math., 74 (1952), 389-400.

5. E. E. Kummer, De generali quadam equatione differentiali tertii ordinis, J. Reine Angew. Math. 100 (1887), 1-9; (a reprint of an article first published in 1834).

6. H. Onose, Oscillatory property of certain nonlinear ordinary differential equations, SIAM. J. Appl. Math. 18 (1970), 715-719.

7. M. Rab, Kriterien für die Oszillation der Lösungen der Differenzialgleichung $\left[p(x) y^{\prime}\right]^{\prime}+g(x) y=0$, Ĉas. Pest Mat., 84 (1959), 335-370. Errata, Ibid., (1960), 91.

8. A. Wintner, A criterion of oscillatory stability, Quart. Appl. Math., 6 (1948), 183185.

9. J. S. W. Wong, A note on second order non-linear oscillation, SIAM Rev., 10 (1968), 88-91.

10. - Oscillation and non-oscillation of second order linear differential equations with integrable coefficients, Trans. Amer. Math. Soc., 144 (1969), 197-216.

11. M. Yelchin, Sur le condition pour q'une solution d'un systeme lineare du second ordre possède deux zeros, Dokl, Akad. Nauk SSR, 51 (1946), 573-576.

12. A. F. Zubova, On oscillation of solutions of a second order equation, Vestn. Leningrad. Univ., 12 (1957), 168-174.

Received March 8, 1971

Texas Tech University 



\section{PACIFIC JOURNAL OF MATHEMATICS}

\section{EDITORS}

H. SAMELSON

Stanford University

Stanford, California 94305

C. R. HobBY

University of Washington

Seattle, Washington 98105
J. DUGUNDJI

Department of Mathematics

University of Southern California

Los Angeles, California 90007

RICHARD ARENS

University of California

Los Angeles, California 90024

\section{ASSOCIATE EDITORS}

E. F. BECKENBACH

B. H. NeumanN

F WoLF

K. YoshidA

\section{SUPPORTING INSTITUTIONS}

UNIVERSITY OF BRITISH COLUMBIA

UNIVERSITY OF SOUTHERN CALIFORNIA

CALIFORNIA INSTITUTE OF TECHNOLOGY

UNIVERSITY OF CALIFORNIA

MONTANA STATE UNIVERSITY

STANFORD UNIVERSITY

UNIVERSITY OF NEVADA

NEW MEXICO STATE UNIVERSITY

OREGON STATE UNIVERSITY

UNIVERSITY OF OREGON

OSAKA UNIVERSITY

UNIVERSITY OF TOKYO

UNIVERSITY OF UTAH

WASHINGTON STATE UNIVERSITY

UNIVERSITY OF WASHINGTON

$\stackrel{*}{*} \stackrel{*}{*} \stackrel{*}{*}{ }^{*}{ }^{*}$ MMEICAN MATHEMATICAL SOCIETY

NAVAL WEAPONS CENTER

Printed in Japan by International Academic Printing Co., Ltd., Tokyo, Japan 


\section{Pacific Journal of Mathematics}

\section{Vol. 42, No. $1 \quad$ January, 1972}

Tage Bai Andersen, On Banach space valued extensions from split faces ........

David Marion Arnold, A duality for quotient divisible abelian groups of finite

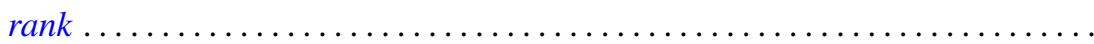

Donald Pollard Ballou, Shock sets for first order nonlinear hyperbolic

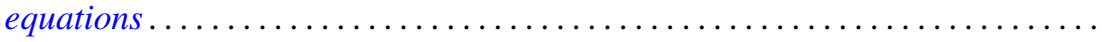

Leon Brown and Lowell J. Hansen, On the range sets of $H^{p}$ functions .........

Alexander Munro Davie and Arne Stray, Interpolation sets for analytic

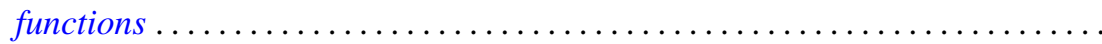

M. G. Deshpande, Structure of right subdirectly irreducible rings. II . . . . . . . . .

Barry J. Gardner, Some closure properties for torsion classes of abelian

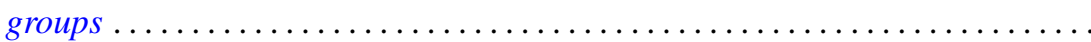

Paul Daniel Hill, Primary groups whose subgroups of smaller cardinality are

direct sums of cyclic groups . . . . . . . . . . . . . . . . . . .

Richard Allan Holzsager, When certain natural maps are equivalences .........

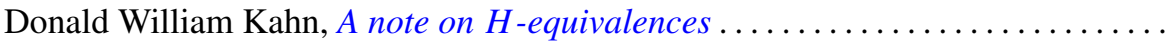

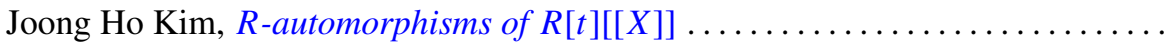

Shin'ichi Kinoshita, On elementary ideals of polyhedra in the 3-sphere.........

Andrew T. Kitchen, Watts cohomology and separability...

Vadim Komkov, A technique for the detection of oscillation of second order

ordinary differential equations .

Charles Philip Lanski and Susan Montgomery, Lie structure of prime rings of characteristic 2

Andrew Lenard, Some remarks on large Toeplitz determinants . .

Kathleen B. Levitz, A characterization of general Z.P.I.-rings. II .

Donald A. Lutz, On the reduction of rank of linear differential systems

David G. Mead, Determinantal ideals, identities, and the Wronskian ...

Arunava Mukherjea, A remark on Tonelli's theorem on integration in product

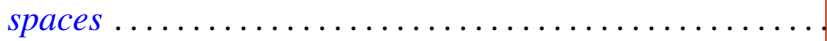

Hyo Chul Myung, A generalization of the prime radical in nonassociative rings.

John Piepenbrink, Rellich densities and an application to unconditionally nonoscillatory elliptic equations.

Michael J. Powers, Lefschetz fixed point theorems for a new class of multi-valued maps .

Aribindi Satyanarayan Rao, On the absolute matrix summability of a Fourier

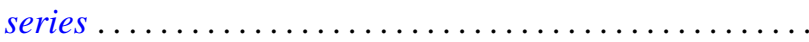

T. S. Ravisankar, On Malcev algebras ......................... 227

William Henry Ruckle, Topologies on sequences spaces . . . . . . . . . . . . . 235

Robert C. Shock, Polynomial rings over finite dimensional rings . . . . . . . . . 251

Richard Tangeman, Strong heredity in radical classes . . . . . . . . . . . . . . 259

B. R. Wenner, Finite-dimensional properties of infinite-dimensional spaces . . . . 267 